\title{
Marine Targets Recognition Through Micro-Motion Estimation from SAR data
}

\author{
Davide Armenise \\ University of Naples \\ darmenise@icloud.com \\ Filippo Biondi \\ University of L'Aquila \\ biopippoo@gmail.com \\ Danilo Orlando \\ Università Degli Studi Niccolò Cusano \\ danilo.orlando@unicusano.it
}

\author{
Pia Addabbo \\ Università Giustino Fortunato \\ p.addabbo@unifortunato.eu
}

\author{
Carmine Clemente \\ University of Strathclyde \\ carmine.clemente@strath.ac.uk
}

\begin{abstract}
The capability to perform Automatic Target Recognition (ATR) from SAR images has great importance for both civilian and military applications. However, this task becomes challenging when the quality and quantity of target information is not sufficient to reliably discriminate the targets. This is particularly important when dealing with marine targets, where features such as scattering intensities and shapes are common to many different targets. This paper investigates the possibility to enhance classification capabilities of marine targets in SAR images by exploiting the micro-motion information. This characterizing source of information, is extracted by applying Doppler sub-apertures and pixel tracking on SAR images containing the target of interest. The proposed approach is validated on real COSMO-SkyMed SAR data demonstrating the effectiveness to discriminate ships through their unique Doppler fingerprint.

Index Terms-Synthetic aperture radar (SAR), micro-motion (m-m), target classification.
\end{abstract}

\section{INTRODUCTION}

The capability to reliably recognize targets in synthetic aperture radar (SAR) images is a topic of great relevance for both civilian and military applications. In particular, marine target surveillance can be performed effectively thanks to the capability of SAR images to be acquired in any weather and light condition. Furthermore, the high contrast features of SAR images of sea and targets allow for reliable detection and segmentation of marine objects. In [1] a vibrating micro-Doppler (m-D) signature for a bistatic SAR system with fixed receiver is analyzed and compared to the signature obtained in a monostatic SAR system. The $\mathrm{m}$-D signature is derived for a vibrating target in the bistatic SAR. The designed model is useful for $\mathrm{m}-\mathrm{D}$ classification. In [2], the authors developed an intrinsic feature of helicopters extracted from m-D signature. In [4], a passive bistatic configuration using a GNSS as illumination in near forward scattering zone for $\mathrm{m}-\mathrm{D}$ analysis is proposed. The study focuses on the case of helicopters rotor blades where the Doppler shift is very high and a relatively large wavelength is useful in reducing the maximum Doppler shift. The theoretical analysis is supported with simulations that demonstrate the effectiveness of the proposed configuration for m-D signature analysis for helicopter rotor blades. In [5] the author present a challenging classification problem of four different radar and sonar systems, consisting of a $40 \mathrm{kHz}$ sonar, a $5.8 \mathrm{GHz}$ wireless pulsed Doppler radar mote, a $10 \mathrm{GHz}$ Xband continuous wave radar, and a $24 \mathrm{GHz} \mathrm{CW}$ radar. Results show that nearly $80 \%$ correct classification can be achieved with $10 \mathrm{~s}$ observations from the $24 \mathrm{GHz} \mathrm{CW}$ radar, whereas $86 \%$ performance can be achieved with $5 \mathrm{~s}$ observations of sonar. In [3], the authors considers feature extraction technique for $\mathrm{m}-\mathrm{D}$ classification and its real-time implementation using a support vector machine classifier on a low-cost, embedded digital signal processor are presented. The effectiveness of the proposed technique is improved through exploitation of the outliers rejection capabilities of robust principal component analysis (PCA) in place of classic PCA. micro-motion (mm) SAR returns can be modeled as sinusoidal frequencymodulated signals in the noise/clutter environment. In [6] a detector based on the generalized likelihood ratio test (GLRT) is proposed to detect such targets using SAR raw signals instead of SAR imagery. The detection performance is derived theoretically and demonstrated by both simulated and quasireal data, showing high detection ability under low signalto-noise/clutter ratios. The approach presented in [12], [13] consists of a novel strategy to estimate the $\mathrm{m}-\mathrm{m}$ of ships from synthetic aperture radar (SAR) images. The predominant vibrational modes of different ships are then estimated. The authors have shown that they can estimate the vibrations of marine targets through pixel tracking techniques while [14] extended the same technique in estimating rivers height. In [7], the vibration signal modeling is deduced and the $\mathrm{m}-\mathrm{m}$ signature of vibrating target is analyzed, based on short-term shift-orthogonal waveform in Multiple input multiple output (MIMO) SAR. The results demonstrate the potential of MIMO SAR for 3D m-m estimation. In [8] authors implement an unsupervised clustering, using all possible extracted features to differentiate ship and non-ship targets. In [9] an approach using Kelvin waves in SAR images is used to estimate marine targets motion parameters. The algorithm considers the polarimetric (Pol) information of SAR images, evaluating a multiple-channel and dual-stage Pol low-rank plus sparse decomposition assisted by Radon transform for clutter reduction, sparse object detection, precise wake inclination estimation, and targets classification. In recent years, many works based on artificial intelligence and deep learning have been investigated. 
In [10] a modified Alexnet structure has been used to design marine targets classification on the Gaofen-3 spaceborne SAR images, achieving an accuracy of $92.10 \%$ in classifying six kinds of targets, with performance is superior compared with other convolutional neural networks (CNNs) and traditional support vector machine algorithms. A full work-flow for SAR marine targets detection and classification on TerraSAR-X high-resolution images is supported in [11], using CNNs and showing that the proposed method is able to perform marine target classification in SAR images.

In this paper we extend the approach proposed in [12], [13] to show that the vibrational features can be used to enhance target recognition of ships. We divide the experiments into four case studies of four different ships. Experiments show that it is possible to discriminate the targets because each of them offers a different vibrational signature, even if the ships present similar physical structures (size, shape). The outline of this paper is the following, Section II introduces the computational model (subsection II-A) and the estimation procedure (subsection II-B) while in section III the experimental results are reported.

\section{Computational Model And Estimation PROCEDURE}

This section consists of two parts aimed at guiding the reader towards the design of the estimation procedure. More precisely, the first part provides a description of the underlying vibrational model from the physical point of view, whereas the second part explains the ideas behind the estimation procedure, presenting the entire processing chain and describing each operation performed in the course of the parameter estimation.

\section{A. Computational Model}

In this subsection, we present the vibrational model generated by a ship. Since the ship is considered as a complex body consisting of several interacting mechanical components, the analysis of its behavior represents a complex task. A common practice consists in firstly dividing the ship into the following components:

- Keel;

- Main structural substructures;

- Local structural elements;

- Electrical power facilities;

- Propulsion systems.

Figure 1 (b) represents the same ship observed in SAR coordinates. The point $\mathrm{P} 1$ is hit by the electromagnetic pulses and, during the image formation process, is projected on the point P2 located on the layovered projection plane constituting the SAR image. If P1 vibrates along the three Euclidean dimensions, P2 exhibits a vibration in the range-azimuth SAR plane located into the new vector-space slant coordinates. The forces involved along the 3-dimensional reference system can be seen in Figure 2 (b). The forces F1, F2 and F3 are generated from the rotation movement of the propulsion propeller, amplified by the sail effect generated by the rudder. The forces F4 and F5 are instead due to the movement of the main axis of the motion transmission generated by the engines. The coupling system and the engine revolutions-perminute (rpm) reduction box generates the force F6 while the Diesel engine will pull the forces F7 and F8. Standard SARprocessing methods are based upon the assumption of a static scene. If targets are moving, their positions in the SAR-image are modified according to the following scheme:

- Fixed azimuth shift from constant range velocity;

- Azimuth defocussing from range acceleration;

- Azimuth defocussing from constant azimuth velocity;

- Azimuth defocussing from azimuth acceleration.

We focus our attention on the Figure 2 where the sensor travels on a straight line with speed $V$ and total acquisition time $T$. The target $P_{1}$ located at the "zero-Doppler" is moving with range and azimuth velocity $\left(v_{c}, v_{D}\right)$ and acceleration components $\left(a_{c}, a_{D}\right)$. At a generic instant between the azimuth sampling, the vector position is equal to:

$$
\left|R_{c, D}(t)\right|=\sqrt{\left[R_{0}-G_{1}\right]^{2}+\left[V t-G_{2}\right]^{2}}
$$

with $G_{1}=\frac{1}{2} a_{c} t^{2}+v_{c} t, G_{2}=\frac{1}{2} a_{D} t^{2}+v_{D} t$ and $\left[R_{0}-G_{1}\right] \gg$ $\left[V t-G_{2}\right]$. Considering the first two terms truncation Taylor series $(1+x)^{\gamma} \approx 1+\gamma x$ and with $V T+v_{D} t \gg \frac{1}{2} a_{D} t^{2}$ and $R_{0} \gg v_{c} t+\frac{1}{2} a_{c} t^{2}$, (1) can be indicated as:

$\left|R_{c, D}(t)\right| \approx R_{0}-v_{c} t+\left[\left(V-v_{D}\right)^{2}-R_{0} a_{c}\right] \frac{t^{2}}{2 R_{0}}, t \in T$.

The above equation can be expressed as follows:

$\left|R_{c, D}(x)\right| \approx R_{0}-\xi_{c_{1}} x+\left[\left(1-\xi_{D}\right)^{2}-\xi_{c_{2}}\right] \frac{x^{2}}{2 R_{0}}, x \in V T$,

we estimate the following motion coefficients:

- $\xi_{c_{1}}=\frac{v_{c}}{V}$ proportional to the range velocity;

- $\xi_{c_{2}}=\frac{a_{c} R_{0}}{V^{2}}$ proportional to the range acceleration;

- $\xi_{D}=\frac{v_{D}}{V}$ proportional to the azimuth velocity.

\section{B. Estimation Procedure}

Sub-pixel offset tracking (SPOT) is a relevant technique to measure large-scale displacements in both range and azimuth directions. The technique is complementary to differential interferometric SAR and persistent scatterers interferometry when the radar phase information is unstable. In this paper, we apply the pixel tracking technique to a single stripmap image instead of multi-temporal interferometric images. Specifically, the single observation is divided into Doppler sub-apertures in order to investigate the fastest displacements of moving targets. Note that the acquisition duration is in the order of a few seconds. We focus on estimating vibrations of some specific point of ships and estimate the dynamic displacement of the scatterers during the Doppler sub-aperture history. In accordance with the frequency subdivision strategy depicted in Figure 4, we observe the offset trend by computing the normalized cross correlation once the image is partitioned into small patches. The estimation procedure consists in shifting the master for each Doppler event and calculating the correlation between adjacent Doppler sub-apertures, according to a smallfrequency baseline strategy. The output of the two-dimensional 
matched filter of the receiver chain can be expressed in a matrix whose elements are as follows [15]:

$$
\begin{aligned}
r_{c, D}^{i, j}= & \frac{A_{c}^{i, j} \sin \left(n_{c} \cdot \delta_{R_{c}} / B_{c}\right)}{\left(n_{c} \cdot \delta_{R_{c}} / B_{c}\right)} \\
& \times \frac{A_{D}^{i, j} \sin \left(n_{D} \cdot \delta_{R_{D}} / B_{D}\right)}{\left(n_{D} \cdot \delta_{R_{D}} / B_{D}\right)} \\
& \times \exp \left(j \frac{4 \pi}{\lambda} R_{c, D}\left(\xi_{c_{1}}, \xi_{c_{2}}, \xi_{D}\right)\right) \\
& n_{c}=0, \ldots, N_{c}-1, n_{D}=0, \ldots, N_{D}-1, \\
& i, j=1, \ldots,\left(G_{D}, G_{t}\right) .
\end{aligned}
$$

Formula (4) is the response of the 2-dimensional matched filter of the point-target $P_{1}$ represented in Figure 2. For each interferometric complex indexed by $i$ and belonging to the particular frequency series of length $G_{D}$ and $j$ belonging to the particular temporal series of length $G_{t}$, the parameters $\delta_{R_{c_{i}}}$ and $\delta_{R_{D}}$ are the chirp and Doppler resolutions respectively, $n_{c}$ and $n_{D}$ is the chirp and Doppler wavenumbers respectively while $B_{c}$ is the bandwidth of the transmitted chirp signal and $B_{D}$ is the synthesized Doppler band. $\left\{A_{c}^{i, j}, A_{D}^{i, j}\right\}$ are the range-Doppler backscattering coefficients. In equation (4) the parameter $R_{c, D}$ is the distance belonging between each chirp-Doppler SAR focused image pixel to the target perturbed by the motion coefficients defined in (3). This means that the center of the adapted filter response consisting of the two-dimensional "sinc" (4) is continuously shifting in space mainly because of the parameters due to the accelerations coefficients $\xi_{c_{2}}, \xi_{D}$ generated by target vibrations. Shifts are subsequently detected by generating several Doppler subapertures. Doppler sub-aperture data will necessarily have to be co-registered ${ }^{1}$. The offset components of the sub-pixel normalized cross-correlation, according to [13] and [14] are described by the complex parameter $\mathbf{D}_{\text {tot }}^{i, j, D)}$ referring to the total displacement, which is estimated by the following equation:

$$
\begin{aligned}
\mathbf{D}_{\text {tot }_{(c, D)}}^{i, j} & =\mathbf{D}_{\operatorname{displ}_{(c, D)}}^{i, j}+\mathbf{D}_{\text {topo }_{(c, D)}}^{i, j}+\mathbf{D}_{\text {orbit }_{(c, D)}}^{i, j} \\
& +\mathbf{D}_{\operatorname{control}_{(c, D)}}^{i, j}+\mathbf{D}_{\text {atmosphere }_{(c, D)}}^{i, j}+\mathbf{D}_{\text {noise }_{(c, D)}}^{i, j}, \\
& i, j=1 \ldots\left(G_{D}, G_{t}\right), \text { where: }
\end{aligned}
$$

- $\mathbf{D}_{\operatorname{displ}_{(c, D)}}^{i, j}$ is the offset component of the signal position presented in (4), generated by the bridge vibrational trend and detected as a sub-pixel misalignment existing between the first SAR image (master) and the $i-t h$ slave Doppler sub-aperture. The shifts are due to $\left(\xi_{c_{1}}, \xi_{c_{2}}, \xi_{D}\right)$ motion coefficients (3);

- $\mathbf{D}_{\text {topo }}^{i, j, D)}$ is the offset component generated by the earth displacement when located on highly sloped terrain;

- $\mathbf{D}_{\text {orbit }_{(c, D)}}^{i, j}$ is the offset caused by residual errors of the satellite orbits;

\footnotetext{
${ }^{1}$ The coregistration process consists to align the pixels of any slave image to the corresponding pixels of the master image. The alignment process is very precise performed at the sub-pixel level.
}

- $\mathbf{D}_{\text {control }}^{i, j, D)}$ is the offset component generated by general attitude and control errors of the flying satellite trajectory; - $\mathbf{D}_{\text {atmosphere }_{(c, D)}^{i, j}}$ and $\mathbf{D}_{\text {noise }}^{i, c, D)}$ are the contributions generated by the electromagnetic aberrations due to atmosphere parameters space and time variations and general disturbances due to thermal and quantization noise, respectively.

Equation (5) is a general case where displacement exists in both range-azimuth dimensions and evolving in time. In the herein considered case, the displacement generated by the engines vibrations, when perturbed by the persistent rough sea, can be significantly greater with respect to any other sporadic displacements due to physical phenomena. With the above remarks in mind, we assume that the main contribution of $\mathbf{D}_{\text {tot }_{(c, D)}}^{i, j}$ is due to the range/azimuth components. Figure 3 is a schematic representation of the parameters estimated by the coregistration procedure. The square number 1 is a focused pixel of the master image and the square number 2 is the same pixel but located on the slave image. The parameters $\mathbf{D}_{\text {tot }_{c}}^{i, j}$ and $\theta^{i, j}$ are the distance between the master and slave pixel centers and the angle respect to the horizontal axis respectively. In the present case, since the shift due to vibrations occurs on the range-azimuth plane, the parameter $\theta^{i, j}$ is an harmonic parameter. Figure 4 represents the frequency allocation plane strategy used to estimate the $\mathrm{m}-\mathrm{m}$. The $d_{b}$ parameter represents the value of the frequency baseline used. The estimated shifts $\mathbf{D}_{\text {tot }(c, D)}^{i, j}$ give the contribution in estimating the vibrational trend oner the space, time and frequency. Figure 5 illustrates the processing framework used to estimate the $\mathrm{m}-\mathrm{m}$ of ships. The model requires only one image in raw format (computational block 1). From this image is calculated its 2D digital Fourier transform (DFT) (computational block 2) which is transferred to computational block 3. This step implements a multi-stage bandpass filter, in order to form multiple SAR images with lower spatial Doppler resolution but containing the information of the infra chromatic shifts. The computational block 4 performs the calculation of the inverse digital Fourier transform (IDFT) and, each raw subproduct will be subsequently focused by the computational block 5. These raw data are coregistered from computational block 6, range-azimuth shifts are extracted from block 7 by two-dimensional cross-correlation and the output of block 7 is a vibrational profile that can be used as feature to perform the target classification as it is or integrated to other features.

TABLE I

EXPERIMENTAL RESULTS OUTLINE

\begin{tabular}{|c|c|c|c|c|}
\hline \hline Case & Ship name & AIS Record & Speed (knots) & Heading $\left(^{\circ}\right.$ ) \\
\hline \hline 1 & Vento di Bora & 271001018 & 18.3 & 290.2 \\
2 & MSC Fantasia & 370648000 & 14.2 & 321.1 \\
3 & BBC London & 305690000 & 10.8 & 163.0 \\
4 & Eurocargo Roma & 247241900 & 16.9 & 144.7 \\
\hline
\end{tabular}


TABLE II

LIST OF SAR ACQUISITIONS

\begin{tabular}{|c|c|}
\hline \hline Case & SAR Image Identification \\
\hline \hline 1 & HI-01-HH-RA-FM-20110209050544-44.2975N-009.1856E.h5 \\
2 & HI-01-HH-RA-FM-20110108050606-44.3069N-009.1815E.h5 \\
3 & HI-01-HH-RA-FM-20110108050606-44.3069N-009.1815E.h5 \\
4 & HI-01-HH-RA-FM-20110108050606-44.3069N-009.1815E.h5 \\
\hline
\end{tabular}

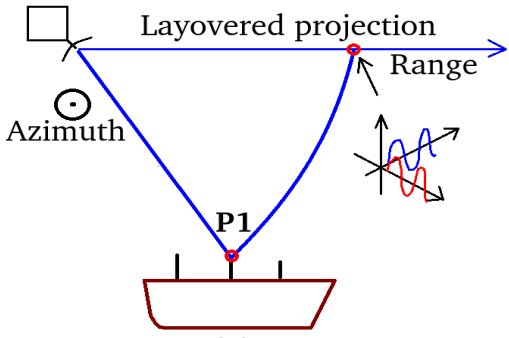

(a)

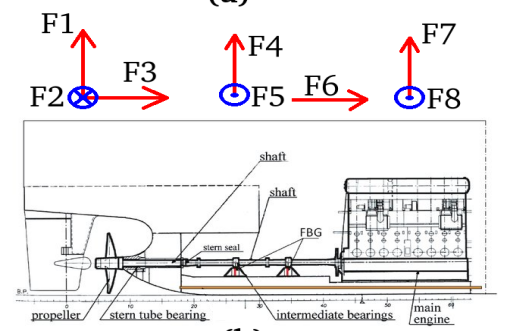

(b)

Fig. 1. (a):representation into the range-azimuth SAR slant coordinates. (b):General scheme observed in the 3D space.

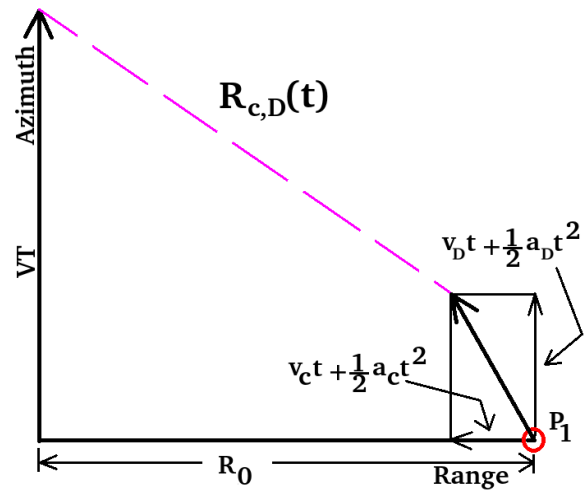

Fig. 2. Moving target radar geometry in the slant-range plane.

\section{EXPERIMENTAL RESULTS}

The experimental results consist of four case studies concerning four different vessels. Ground truth information for each ship is obtained from automatic information system (AIS) data. All target information is outlined in the Table I. Table II reports the identification numbers of the SAR data used for all the experiments. The case of study 1 consists in a Maltese container ship where the spatial distribution Doppler spectral synthesis is depicted in Figure 6. The case study number 2 consists of the MSC Fantasia which is a cruise passenger ship, built in 2008 and currently sailing under the Panama

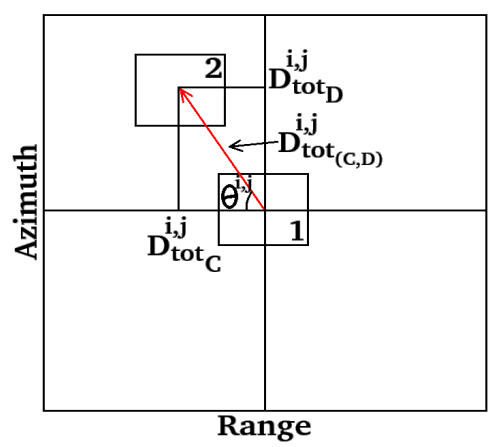

Fig. 3. Schematic representation of isolated pixels with a certain shift due to space displacement.

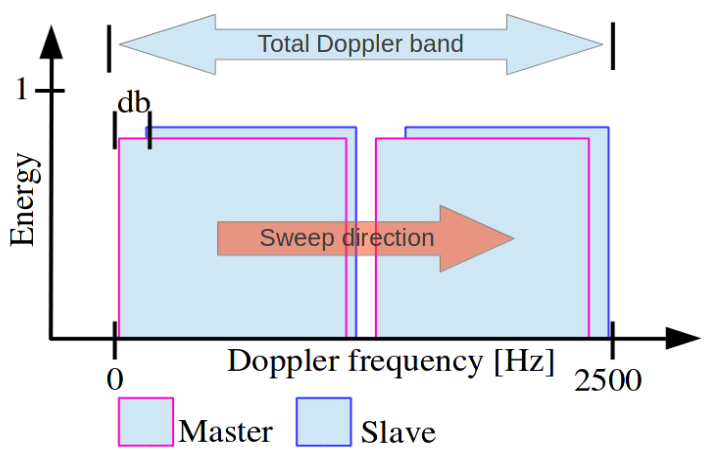

Fig. 4. Frequebcy allocation plane.

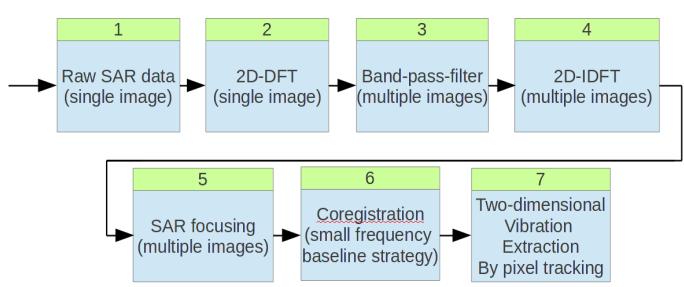

Fig. 5. Computational scheme.

flag. The spatial distribution Doppler spectral synthesis is shown in Figure 7. The BBC London is a General Cargo ship built in 2011 that currently sails under the Antigua and Barbuda flag. Its Doppler spectral synthesis distribution is shown in Figure 8. The Eurocargo Roma is a Cargo Ship, built in 2010 which currently sails under the flag of Italy. The Doppler spectral synthesis of Eurocargo Roma is represented in Figure 9. Figures 10-13 (a) show the optical images of the 4 ships of interest while Figures 10-13 (b) shows the vibrational keel graphs of each ship obtained from the Doppler spectra synthesis result. In particular, the keel graph is computed as the average normalized energy along the longitudinal axis of the ship. From the derived keel graphs it can be appreciated how this feature is characterizing and has the a discriminating potential. In order to give a quantitative assessment of the discriminating potential we present in Table III the cross- 


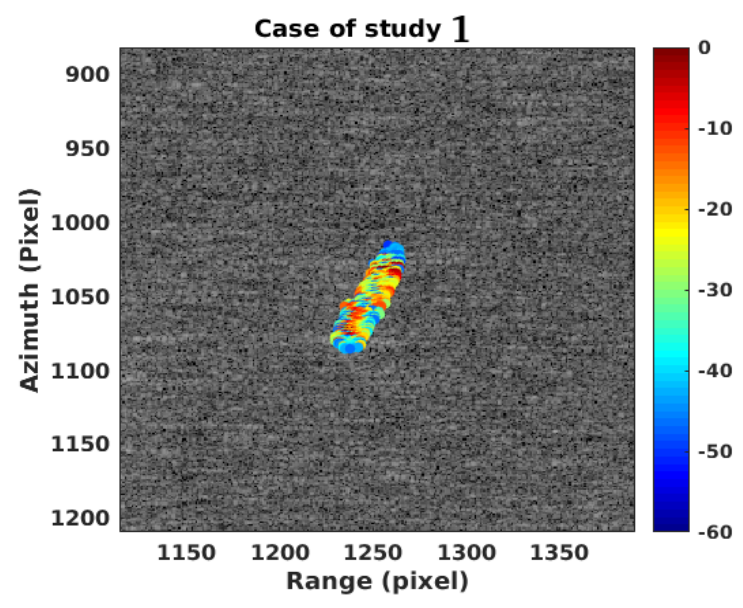

Fig. 6. Case of study 1 spatial distribution Doppler spectral synthesis.

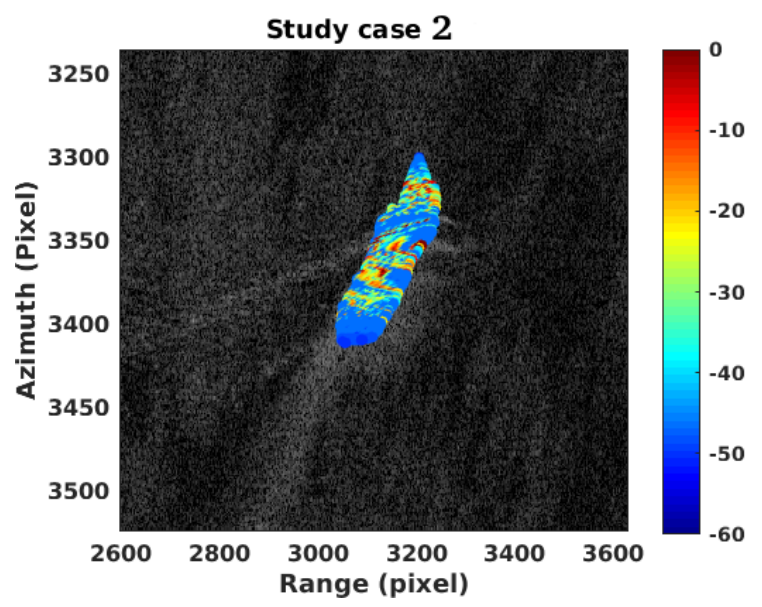

Fig. 7. Case of study two spatial distribution Doppler spectral synthesis.

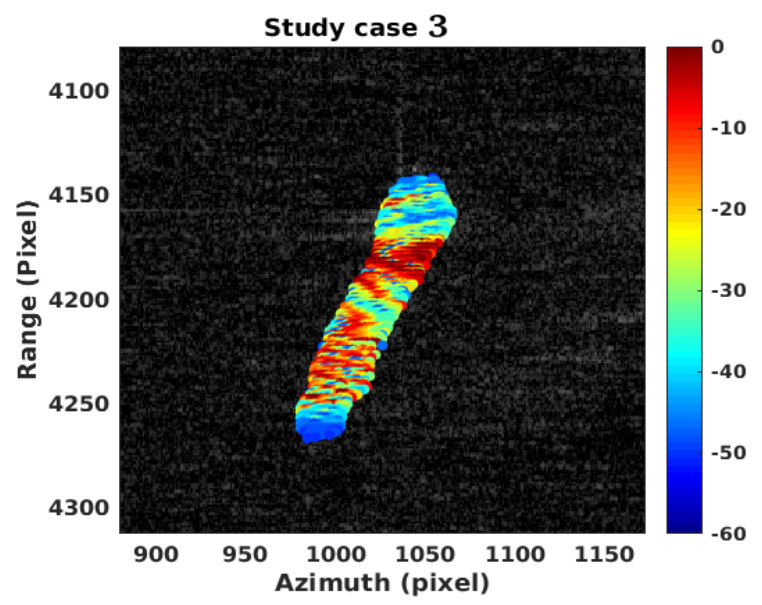

Fig. 8. Case of study 3 spatial distribution Doppler spectral synthesis.

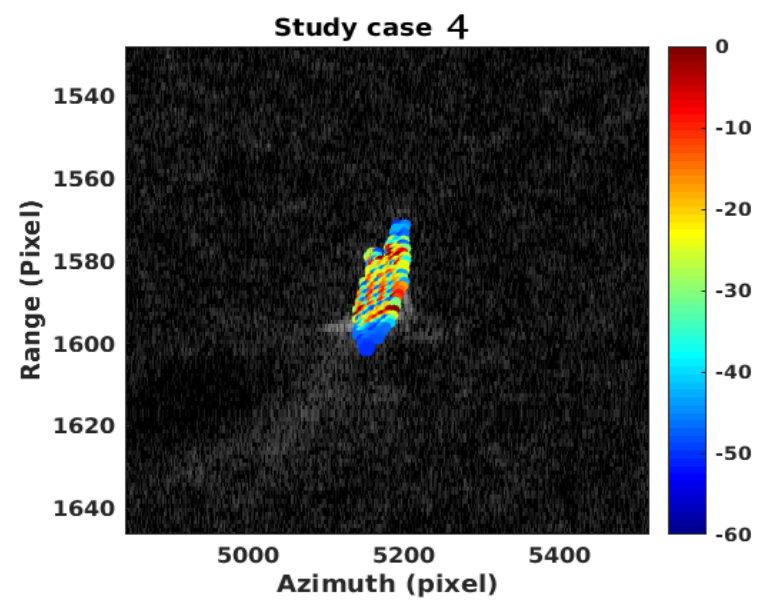

Fig. 9. Case of study four spatial distribution Doppler spectral synthesis.

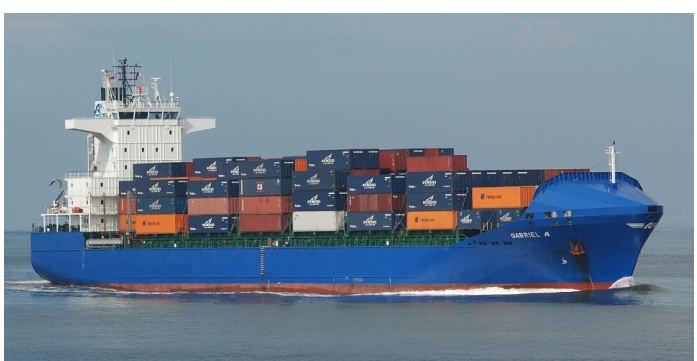

(a)

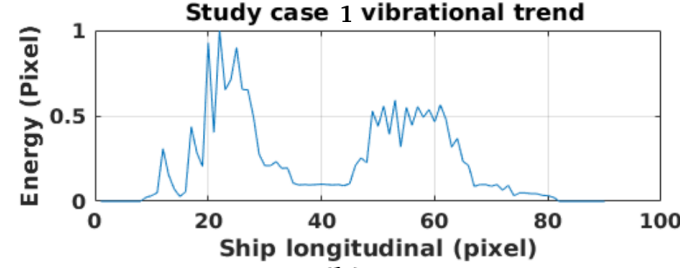

(b)

Fig. 10. (a): Case of study 1 optical image. (b): keel line vibrational signature.

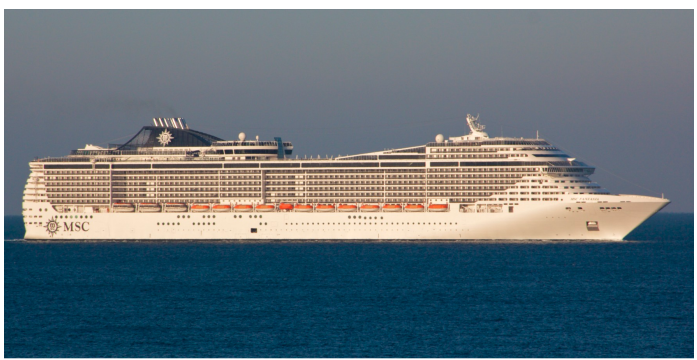

(a)

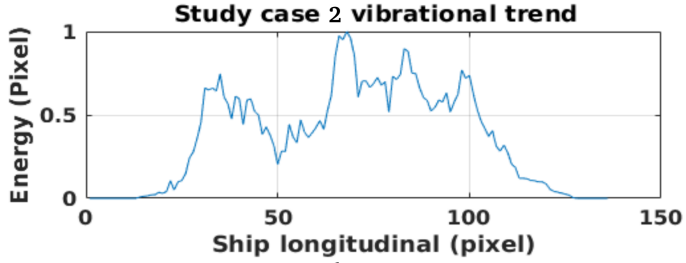

(b)

Fig. 11. (a): Case of study 2 optical image. (b): keel line vibrational signature. 


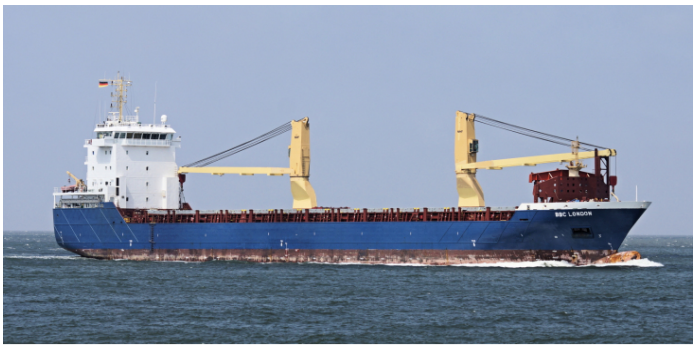

(a)

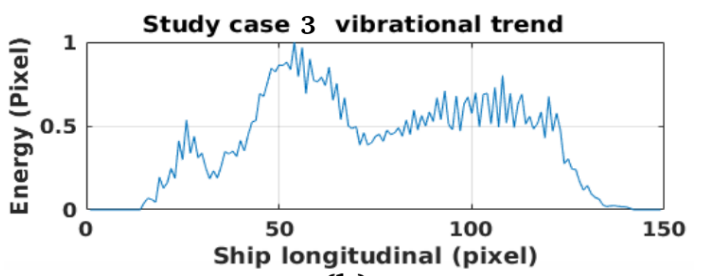

(b)

Fig. 12. (a): Case of study 3 optical image. (b): keel line vibrational signature.

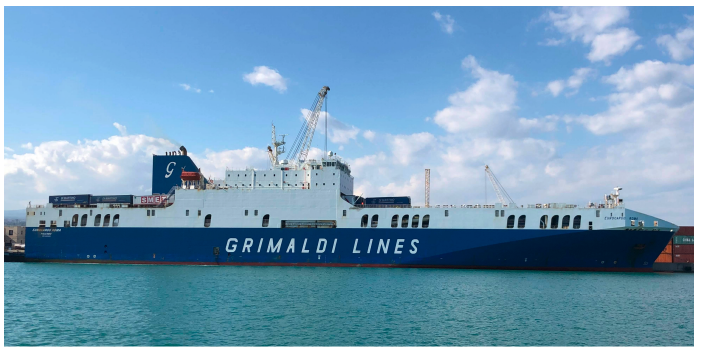

(a)

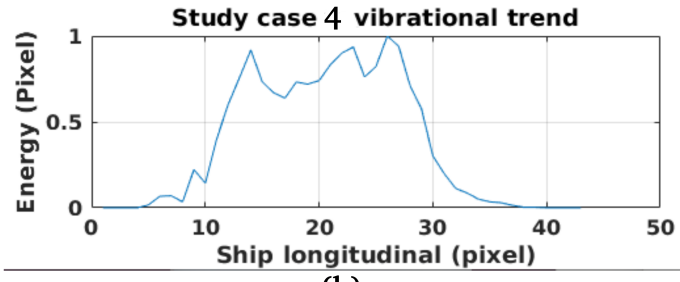

(b)

Fig. 13. (a): Case of study 4 optical image. (b): keel line vibrational signature.

correlation coefficients between the 4 keel graphs obtained. The cross-correlation coefficients are below 0.5 when two different ships are considered, in particular the cross-correlation coefficient between the vibrational keel graphs of cases 2 and 3 reaches 0.4250 which is still a relatively low value and that would allow even a simple classifier to perform a correct target recognition.

\section{CONCLUSIONS}

In this paper we investigated the possibility of reinforcing the classification performance of marine radar targets that are often available at low resolution. We have estimate a new communication channel generated by the vibration of targets that offer different vibration profiles from ship to ship. The proposed approach has been tested on real SAR data from COSMO-SkyMed data and has shown that a novel family of targets features. The novel Doppler fingerprint can be than used as stand-alone or complementary feature to strengthen the performance of the marine AIS framework.

TABLE III

CRoss-CORRELATION COEFFICIENTS

\begin{tabular}{|c|c|c|c|c|}
\hline \hline Case & $\mathbf{1}$ & $\mathbf{2}$ & $\mathbf{3}$ & $\mathbf{4}$ \\
\hline \hline 1 & 1.0000 & 0.2326 & 0.2528 & 0.3268 \\
2 & 0.3059 & 1.0000 & 0.4250 & 0.2163 \\
3 & 0.2528 & 0.4250 & 1.0000 & 0.1882 \\
4 & 0.3268 & 0.2163 & 0.1882 & 1.0000 \\
\hline
\end{tabular}

\section{REFERENCES}

[1] C. Clemente and J. J. Soraghan, "Vibrating Target Micro-Doppler Signature in Bistatic SAR With a Fixed Receiver," in IEEE Transactions on Geoscience and Remote Sensing, vol. 50, no. 8, pp. 3219-3227, Aug. 2012, doi: 10.1109/TGRS.2011.2180394.

[2] R. Zhang, G. Li, C. Clemente and J. J. Soraghan, "Multi-aspect microDoppler signatures for attitude-independent L/N quotient estimation and its application to helicopter classification," in IET Radar, Sonar and Navigation, vol. 11, no. 4, pp. 701-708, 4 2017, doi: 10.1049/ietrsn.2016.0271.

[3] J. Zabalza, C. Clemente, G. Di Caterina, Jinchang Ren, J. J. Soraghan and S. Marshall, "Robust PCA micro-doppler classification using SVM on embedded systems," in IEEE Transactions on Aerospace and Electronic Systems, vol. 50, no. 3, pp. 2304-2310, July 2014, doi: 10.1109/TAES.2014.130082.

[4] C. Clemente and J. J. Soraghan, "GNSS-Based Passive Bistatic Radar for Micro-Doppler Analysis of Helicopter Rotor Blades," in IEEE Transactions on Aerospace and Electronic Systems, vol. 50, no. 1, pp. 491-500, January 2014, doi: 10.1109/TAES.2013.120018.

[5] S. Z. Gurbuz, C. Clemente, A. Balleri and J. J. Soraghan, "MicroDoppler-based in-home aided and unaided walking recognition with multiple radar and sonar systems," in IET Radar, Sonar and Navigation, vol. 11, no. 1, pp. 107-115, 1 2017, doi: 10.1049/iet-rsn.2016.0055.

[6] B. Deng, H. -. Wang, X. Li, Y. -. Qin and J. -. Wang, "Generalised likelihood ratio test detector for micro motion targets in synthetic aperture radar raw signals," in IET Radar, Sonar and Navigation, vol. 5, no. 5, pp. 528-535, June 2011.

[7] G. Zhao, Y. Fu, L. Nie and Z. Zhuang, "Imaging and micro-Doppler analysis of vibrating target in multi-inputmulti-output synthetic aperture radar," in IET Radar, Sonar Navigation, vol. 9, no. 9, pp. 1360-1365, 12 2015, doi: 10.1049/iet-rsn.2014.0480.

[8] W. Ao, F. Xu, Y. Qian and Q. Guo, "Feature clustering based discrimination of ship targets for SAR images," in The Journal of Engineering, vol. 2019, no. 20, pp. 6920-6922, 102019.

[9] F. Biondi, "A Polarimetric Extension of Low-Rank Plus Sparse Decomposition and Radon Transform for Ship Wake Detection in Synthetic Aperture Radar Images," in IEEE Geoscience and Remote Sensing Letters, vol. 16, no. 1, pp. 75-79, Jan. 2019.

[10] M. Ma, H. Zhang, X. Sun and J. Chen, "Maritime targets classification based on CNN using Gaofen-3 SAR images," in The Journal of Engineering, vol. 2019, no. 21, pp. 7843-7846, 112019.

[11] C. Bentes, D. Velotto and B. Tings, "Ship Classification in TerraSAR$\mathrm{X}$ Images With Convolutional Neural Networks," in IEEE Journal of Oceanic Engineering, vol. 43, no. 1, pp. 258-266, Jan. 2018.

[12] F. Biondi, "COSMO-SkyMed Staring Spotlight SAR Data for MicroMotion and Inclination Angle Estimation of Ships by Pixel Tracking and Convex Optimization," in Remote Sens. 2019, 11, 766.

[13] F. Biondi, P. Addabbo, D. Orlando, and C. Clemente, "Micro-motion estimation of maritime targets using pixel tracking in COSMO-SkyMed synthetic aperture radar dataAn operative assessment," in Remote Sensing, 11(14), pp. 1637, (2019).

[14] F. Biondi, A. Tarpanelli, P. Addabbo, C. Clemente, and D. Orlando, "Pixel Tracking to Estimate Rivers Water Flow Elevation Using CosmoSkyMed Synthetic Aperture Radar Data," in Remote Sensing, 11(21), 2574, 2019.

[15] M. A. Richards, "Fundamentals of radar signal processing. Tata McGraw-Hill Education,” (2005). 